\title{
Effect of Omega-3 and Korean Red Ginseng on Children with Attention Deficit Hyperactivity Disorder: An Open-Label Pilot Study
}

\author{
Jeewon Lee', Areum Lee', Ji-Hoon Kim², Yun Mi Shin ${ }^{3}$, Seong-Ju Kim³ ${ }^{3}$ Woo Dong $\mathrm{Cho}^{3}$, Soyoung Irene Lee ${ }^{1}$ \\ ${ }^{1}$ Department of Psychiatry, Soonchunhyang University Bucheon Hospital, Bucheon, ${ }^{2}$ Department of Psychiatry, Pusan National University \\ Yangsan Hospital, Yangsan, ${ }^{3}$ Department of Psychiatry and Behavioral Sciences, Ajou University Hospital, Suwon, Korea
}

\begin{abstract}
Objective: The purpose of the present study was to evaluate the efficacy of omega- 3 and Korean red ginseng on improving attention deficit hyperactivity disorder (ADHD) symptoms and cognitive function in children with ADHD.

Methods: A total of 40 children aged 6 to 12 years diagnosed with ADHD participated in this open-label trial. Participants received daily supplements containing $500 \mathrm{mg}$ of omega-3 (eicosapentaenoic acid, $294 \mathrm{mg}$; docosahexaenoic acid, $206 \mathrm{mg}$ ) and $3 \mathrm{mg}$ of Korean red ginseng extract (combination of ginsenoside Rg1, Rb1, and Rg3) for 12 weeks. No psychotropic drug was allowed during the study period. ADHD symptoms were assessed using the ADHD Rating Scale (ADHD-RS) and Clinical Global Impression-Severity (CGI-S) scale. Neuropsychological tests on sustained attention, short-term memory, and executive function were also assessed.

Results: After 12 weeks, participants showed significant improvements on ADHD-RS (31.12 \pm 8.82 at baseline, $24.15 \pm$ 11.45 at endpoint; $p<0.001)$ and CGI-S $(3.38 \pm 1.18$ at baseline, $2.94 \pm 1.00$ at endpoint; $p<0.001)$. On the Continuous Performance Test, commission errors significantly decreased, while reaction time significantly increased. Immediate recall and delayed recall on both Auditory Verbal Learning Test and Complex Figure Test showed significant improvements. Scores of Color-Word Task from Stroop Color-Word Test also showed significant improvements after the treatment. The supplement was well tolerated.

Conclusion: The results of this pilot study suggest that the combination of omega-3 and Korean red ginseng may improve ADHD symptoms and cognitive function including attention, memory, and executive function in children with ADHD. Future randomized placebo-controlled trials with a larger sample is warranted.
\end{abstract}

KEY WORDS: Attention deficit disorder with hyperactivity; Child; Omega-3; Ginseng.

\section{INTRODUCTION}

Attention deficit hyperactivity disorder (ADHD) is a neurodevelopmental disorder marked by ongoing symptoms of inattention, hyperactivity and impulsivity, which interferes with development and functioning in multiple cognitive domains, including attention, memory, and executive function [1]. Although psychostimulants for ADHD are known for its high efficacy, many parents are still reluctant in electing pharmacotherapies for their ADHD children due to concerns of side effects, especially for

Received: February 27, 2019/ Revised: April 21, 2019

Accepted: April 24, 2019

Address for correspondence: Soyoung Irene Lee

Department of Psychiatry, Soonchunhyang University Bucheon

Hospital, 170 Jomaru-ro, Wonmi-gu, Bucheon 14584, Korea

E-mail: irenelee@schmc.ac.kr

ORCID: https://orcid.org/0000-0003-2473-2954 those with relatively moderate symptoms $[2,3]$.

Omega-3 polyunsaturated fatty acids (PUFAs), including eicosapentaenoic acid (EPA) and docosahexaenoic acid (DHA) are essential nutrients that provide a range of neurobiological activities via anti-inflammation, anti-oxidation, neuroplasticity, and modulation of neurotransmitters. Many studies have reported that omega-3 has beneficial effects on mood and anxiety disorders [4]. It also has been reported that children with ADHD have low levels of omega-3 PUFAs and that these correlated significantly with ADHD symptoms [5]. Many clinical trials have been performed to evaluate the effects of the omega-3 supplementation in ADHD children, suggesting significant, but small inconsistent results [6-8].

Ginsenosides, a saponin constituents of the Korean red ginseng (Panax ginseng), have been reported to have various effects on the central nervous system [9]. Rodent mod-

(ㄷ) This is an Open-Access article distributed under the terms of the Creative Commons Attribution Non-Commercial License (http://creativecommons.org/licenses/by-nc/4.0) which permits unrestricted non-commercial use, distribution, and reproduction in any medium, provided the original work is properly cited. 
els showed that ginsenosides can facilitate the release of acetylcholine especially in the hippocampus and increase dopamine and norepinephrine concentrations in the cerebral cortex $[10,11]$. The neurotrophic and neuroprotective properties of ginsenosides have also been suggested [12]. While much more research needs to be done on the effects of Korean red ginseng on ADHD, a recent randomized placebo-controlled study showed that Korean red ginseng significantly improves ADHD symptoms in children [13].

Given the significant but relatively small and limited effect of omega-3 on ADHD, combining another type of well-tolerated dietary supplement with a potential effect on ADHD such as Korean red ginseng may produce beneficial effects. The present pilot study aimed to investigate the efficacy of combined omega-3 and Korean red ginseng supplementation on ADHD symptoms and cognitive function in children with ADHD.

\section{METHODS}

\section{Study Design and Participants}

This was a 12-week, open-label pilot study. The study was approved by the Institutional Review Board of Soonchunhyang University Hospital (SCHBC 2016-11-015002). School-aged children between 6 and 12 years with attention problems were recruited for this study through advertisements and clinical referrals from 3 university-based hospitals. Informed consent and written assent were obtained by all parents and children, respectively. The clinical diagnosis of ADHD and comorbidities of the participants were confirmed by a child psychiatrist using the Kiddie Schedule for Affective Disorders and Schizophrenia (K-SADS).

Exclusion criteria included significant psychiatric disorders (depressive disorder, bipolar disorder, psychotic disorder, and Tourette's disorder), developmental disorders (intellectual disability and autism spectrum disorder), neurologic disorders (epilepsy and brain damage), and ongoing chronic medical conditions. Children who had taken psychotropic drugs during the past 3 months preceding inclusion and those in need of emergent pharmacological treatment for ADHD were also excluded.

\section{Study Treatment}

Participants received daily supplements containing
$500 \mathrm{mg}$ of omega-3 (EPA, $294 \mathrm{mg}$; DHA, $206 \mathrm{mg}$ ) and 3 $\mathrm{mg}$ of Korean red ginseng extract (combination of ginsenoside Rg1, Rb1, and Rg3) for 12 weeks. This dosage and EPA/DHA ratio were chosen based on previous studies $[7,13]$. No psychotropic drugs were allowed during the trial. Compliance was assessed by counting the leftover pills that parents returned to the investigators at each visit. Participants who consumed lower than $70 \%$ of assigned pills were considered noncompliant and were withdrawn from the study.

\section{Primary Outcome Measures}

ADHD Rating Scale (ADHD-RS) and Clinical Global Impression-Severity (CGI-S) were assessed at 4 time points: baseline, week 2, week 6 , and week 12 . ADHD-RS is composed 9 items reflecting symptoms of inattention and 9 items reflecting symptoms of hyperactivity and impulsivity [14]. In this study, the clinician administered and scored the validated Korean version of ADHD-RS [15]. CGI-S assesses the clinician's impression of the patient's current illness state on a 7-point scale from 1 (normal, not ill) to 7 (among the most extremely ill patients) [16].

\section{Secondary Outcome Measures}

The following neuropsychological tests examining attention, memory and executive function were performed at baseline and endpoint.

Advanced Test of Attention is a computerized Continuous Performance Test (CPT) developed in Korea to measure a child or adolescent's sustained and selective attention [17]. This test generates age- and sex-adjusted T scores (mean \pm standard deviation, $50 \pm 10$ ) of omission error, commission error, reaction time, and reaction time variability. Scores above 60 are considered borderline and scores above 70 are considered problematic.

Rey's Auditory Verbal Learning Test (AVLT) and Complex Figure Test (CFT), reorganized and standardized for Korean children were used [18]. AVLT assesses verbal learning and memory, while CFT measures visuospatial abilities and visual memory. The scores are presented as age- and sex-adjusted converted score $(10 \pm 3)$. Higher scores indicate better performance.

Stroop Color-Word Test (SCWT) is one of the most widely used measure of executive function which has been modified in numerous versions. The Korean version of SCWT standardized for children from the Kims Execu- 
tive Intelligence Test was used in the study [19]. Time required to complete the 3 tasks - Color, Word, ColorWord-is measured and presented as age- and sex-adjusted converted score $(10 \pm 3)$.

\section{Safety and Tolerability}

The safety and tolerability of the study treatment was assessed by monitoring adverse events, vital signs and physical examination. At each study visit, parents were asked an open-ended question regarding adverse events. The investigator recorded all reported adverse events and judged each event in terms of its relationship to the study treatment.

\section{Statistical Analysis}

Repeated measures ANOVA with Bonferroni's correction were conducted for the analysis of the change in ADHD-RS and CGI-S scores over the 12-week trial. Modified intention-to-treat analysis included participants who started the treatment and received at least one post-baseline assessment. For those who did not complete the full trial, the last ADHD-RS and CGI-S score was carried forward to the 12-week $(n=4)$.

Paired $t$ tests were conducted to compare the baseline and endpoint scores of the neuropsychological test measures. Per-protocol analysis included participants who followed the protocol and completed the entire trial. Intention-to-treat analysis was not employed because the present study was a non-randomized study mainly concerned with the treatment efficacy, and none of the dropped-out participants received post-baseline neuropsychological tests.

Changes in vital signs of the intention-to-treat sample were compared at baseline and endpoint using paired $t$ tests. The comparison of the demographics and the baseline scores of ADHD-RS between the participants who dropped out and those who completed the trial was performed by Mann - Whitney test and Fisher's exact test. Effect sizes were reported using Cohen's d. All analyses were performed with the SPSS Statistics version 20 (IBM Co., Armonk, NY, USA). A $p$ value $<0.05$ was considered statistically significant.

\section{RESULTS}

Forty children (31 boys, 9 girls; mean age, $8.00 \pm 1.45$
Table 1. Baseline characteristics of the participants $(n=40)$

\begin{tabular}{lc}
\hline \multicolumn{1}{c}{ Characteristic } & Data \\
\hline Age $(y r)$ & $8.00 \pm 1.45$ \\
Sex & $31(77.5)$ \\
Male & $9(22.5)$ \\
Female & \\
School year & $11(27.5)$ \\
1st grade & $13(32.5)$ \\
2nd grade & $7(17.5)$ \\
3rd grade & $5(12.5)$ \\
4th grade & $3(7.5)$ \\
5th grade & $1(2.5)$ \\
6th grade & \\
ADHD type & $16(40.0)$ \\
Inattentive & $1(2.5)$ \\
Hyperactive/impulsive & $23(57.5)$ \\
Combined & $31.45 \pm 8.77$ \\
Baseline ADHD-RS & $3.43 \pm 1.20$ \\
Baseline CGI-S &
\end{tabular}

Values are presented as mean \pm standard deviation or number (\%). ADHD, attention deficit hyperactivity disorder; ADHD-RS, ADHDRating Scale; CGI-S, Clinical Global Impressions-Severity.

years) diagnosed with ADHD were enrolled in the study (Table 1). Six children dropped out during the trial: 4 were unable to meet scheduled study visits and 2 were noncompliant. Mean age, gender distribution and baseline scores of ADHD-RS between those who dropped out and those who completed the study didn't show any significant difference. The mean of baseline ADHD-RS score was $31.45 \pm 8.77$, and the mean CGI-S score was $3.43 \pm$ 1.20, which corresponds to 'mild to moderate' ADHD [20].

After 12 weeks, participants showed significant improvements on ADHD-RS $(31.45 \pm 8.77$ at baseline, $24.75 \pm 11.08$ at week 12; $p<0.001)$ and CGI-S (3.43 \pm 1.20 at baseline, $3.00 \pm 1.04$ at week $12 ; p<0.001$ ) (Table 2). Both scores improved significantly 6 weeks after initiation of treatment and continued to improve over the course of the trial.

On the visual CPT, commission errors significantly decreased, while response time significantly increased at endpoint compared to baseline (Table 3). Immediate recall and remote recall on both AVLT and CFT improved significantly. On SCWT, scores of Color-Word Task showed significant improvements.

Tolerance of the supplement was good, with only one case reporting transient headache potentially related to the treatment. Monitored vital signs including blood pressure and pulse rate showed no significant difference. 
Table 2. Change in ADHD-RS and CGI-S scores

\begin{tabular}{lcccccc}
\hline Scale & Baseline & Week 2 & Week 6 & Week 12 & $F$ & ES \\
\hline ADHD-RS & & & & & \\
Total & $31.45 \pm 8.77$ & $28.95 \pm 11.35$ & $26.13 \pm 11.96^{* * *}$ & $24.75 \pm 11.08^{* * *}$ & $20.63^{* * *}$ & 1.45 \\
Inattention & $18.35 \pm 5.20$ & $17.08 \pm 6.40$ & $15.40 \pm 6.81^{* * *}$ & $14.63 \pm 6.52^{* * *}$ & $18.45^{* * *}$ & 1.38 \\
$\quad$ Hyperactivity & $13.10 \pm 6.16$ & $11.88 \pm 6.53^{*}$ & $10.73 \pm 6.42^{* * *}$ & $10.13 \pm 6.09^{* * *}$ & $13.94^{* * *}$ & 1.11 \\
CGI-S & $3.43 \pm 1.20$ & $3.25 \pm 1.03$ & $2.98 \pm 0.97^{* * *}$ & $3.00 \pm 1.04^{* * *}$ & $11.36^{* * *}$ & 1.08 \\
\hline
\end{tabular}

Values are presented as mean \pm standard deviation.

ADHD-RS, Attention Deficit Hyperactivity Disorder-Rating Scale; CGI-S, Clinical Global Impressions-Severity; ES, effect size.

${ }^{*} p<0.05,{ }^{* * *} p<0.001$; in relation to baseline (Bonferroni's post-test).

ES was calculated as Cohen's d (0.8 = large, $0.5=$ medium, and $0.2=$ small $)$.

Table 3. Baseline and endpoint results of neuropsychological tests

\begin{tabular}{|c|c|c|c|c|}
\hline Test & Baseline & Endpoint & $t$ & ES \\
\hline \multicolumn{5}{|l|}{ CPT } \\
\hline \multicolumn{5}{|l|}{ Visual } \\
\hline Omission errors & $73.94 \pm 20.13$ & $76.00 \pm 20.17$ & -0.57 & 0.10 \\
\hline Commission errors & $83.29 \pm 18.57$ & $73.68 \pm 22.25$ & $4.14^{* * *}$ & 0.71 \\
\hline Reaction time & $52.06 \pm 15.07$ & $59.21 \pm 14.80$ & $-3.60^{* * *}$ & 0.62 \\
\hline Reaction time variability & $70.09 \pm 17.51$ & $71.68 \pm 18.11$ & -0.502 & 0.09 \\
\hline \multicolumn{5}{|l|}{ Auditory } \\
\hline Omission errors & $66.79 \pm 21.11$ & $69.74 \pm 21.92$ & -0.90 & 0.15 \\
\hline Commission errors & $70.59 \pm 21.65$ & $65.09 \pm 18.19$ & 2.03 & 0.35 \\
\hline Reaction time & $41.41 \pm 15.90$ & $44.06 \pm 14.04$ & -1.33 & 0.23 \\
\hline Reaction time variability & $50.68 \pm 14.59$ & $52.50 \pm 12.92$ & -0.70 & 0.12 \\
\hline \multicolumn{5}{|l|}{ AVLT } \\
\hline Immediate recall & $10.35 \pm 2.64$ & $12.76 \pm 3.17$ & $-5.01^{* * *}$ & 0.86 \\
\hline Delayed recall & $8.85 \pm 3.10$ & $10.21 \pm 3.56$ & $-2.78^{*}$ & 0.48 \\
\hline Delayed recognition & $10.15 \pm 2.88$ & $9.79 \pm 2.91$ & 0.79 & 0.14 \\
\hline \multicolumn{5}{|l|}{ CFT } \\
\hline Drawing & $8.12 \pm 2.84$ & $8.32 \pm 2.80$ & -0.47 & 0.08 \\
\hline Immediate recall & $8.74 \pm 2.78$ & $10.74 \pm 3.50$ & $-3.50^{* * *}$ & 0.60 \\
\hline Delayed recall & $8.85 \pm 2.90$ & $10.29 \pm 3.65$ & $-2.48^{*}$ & 0.43 \\
\hline \multicolumn{5}{|l|}{ SWCT } \\
\hline Color & $9.24 \pm 3.37$ & $9.35 \pm 3.37$ & -0.268 & 0.05 \\
\hline Word & $8.71 \pm 3.13$ & $9.56 \pm 3.67$ & -1.70 & 0.31 \\
\hline Color-Word & $8.79 \pm 3.40$ & $9.76 \pm 3.02$ & $-2.23^{*}$ & 0.38 \\
\hline
\end{tabular}

Values are presented as mean \pm standard deviation.

ES, effect size; CPT, Continuous Performance Test; AVLT, Auditory Verbal Learning Test; CFT, Complex Figure Test; SWCT, Stroop Color - Word Test. ${ }^{*} p<0.05,{ }^{* *} p<0.01,{ }^{* * *} p<0.001$.

ES was calculated as Cohen's d (0.8 = large, $0.5=$ medium, and $0.2=$ small).

\section{DISCUSSION}

Combination of omega-3 and Korean red ginseng improved symptoms of ADHD in children as demonstrated by reduction in the clinician-administered ADHD-RS and CGI-S scores. Many previous studies on omega-3 have shown significant effects in improving clinical symptoms of ADHD [7]. Still, there are few studies which showed the improvements by neuropsychological tests. The results of the present study showed improvements in several neuro- psychological variables.

First, attention was improved demonstrated by decreased commission errors on the CPT. A previous study of omega-3 on ADHD children showed significant improvement in many variables including commission errors on the CPT [21]. A previous study of red ginseng on ADHD children showed significant difference in omission errors on the CPT [22]. In the present study, the commission error at baseline had the worst score compared to other variables. This may provide explanation for why only the 
commission errors showed improvement. Other variables could have had too little possibility of improvement. Another significant difference on the CPT was the increased response time after the treatment. The response time at baseline and endpoint were both within the standard deviation of mean of the T-score $(50 \pm 10)$. Therefore, it could be suggested that the response time changed within normal limits, implying not much clinical significance.

Second, improvements in short-term memory were found on the AVLT and CFT. Previous studies of omega-3 have failed to show any significant difference in memory variables in ADHD children [23-25]. One possible explanation for such negative results is that psychostimulants modulates higher cortical functions upon activation of the noradrenergic system in the brain, but omega-3 fatty acids, especially DHA may depress central noradrenergic system which has been shown to have aggression-controlling effects [25]. Therefore, ginsenosides' effect on norepinephrine and acetylcholine may have had a role in the improved memory variables in the present study.

Third, improvements in executive function were found, demonstrated by improvement of scores of Color-Word Task found on the SWCT. The Color-Word task especially measures mental flexibility and the ability to inhibit dominant response. The results were consistent with the previous study which showed significant improvement of executive function on the SCWT by omega-3 on Mexican children [23].

The present study has important limitations because of the methodology and small sample size. The study was an open-label study which is vulnerable to observer bias and placebo effect. The lack of control group makes it impossible to determine whether the improvements are due to the treatment or other nonspecific factors. Lastly, it is unclear whether the results of the present study are effects of omega-3, Korean red ginseng, or the combination.

The present study demonstrated a significant improvement in ADHD symptoms and cognitive function including attention, memory and executive function in children with ADHD by combined supplementation of omega-3 fatty acids and Korean red ginseng, which was welltolerated. These findings are preliminary and warrants confirmation in larger, double-blind, randomized, placebo-controlled trials with longer periods. The effect of combining omega-3 and Korean red ginseng, compared to that of omega-3 and Korean red ginseng alone should also be investigated.

\section{- Acknowledgments}

This study was supported by a grant from Metaformula (Seoul, South Korea) and Soonchunhyang University Research Fund.

\section{- Conflicts of Interest}

No potential conflict of interest relevant to this article was reported.

\section{- Author Contributions}

Conceptualization: Jeewon Lee, Ji-Hoon Kim, Yun Mi Shin, Soyoung Irene Lee. Data acquisition: Jeewon Lee, Areum Lee, Ji-Hoon Kim, Yun Mi Shin, Seong-Ju Kim, Woo Dong Cho, Soyoung Irene Lee. Formal analysis: Areum Lee, Jeewon Lee. Funding: Soyoung Irene Lee. Supervision: Soyoung Irene Lee. Writing-original draft: Jeewon Lee. Writing-review \& editing: Jeewon Lee, Soyoung Irene Lee.

\section{ORCID}

Jeewon Lee https://orcid.org/0000-0001-5930-1834

Areum Lee https://orcid.org/0000-0001-6931-8488 Ji-Hoon Kim https://orcid.org/0000-0001-8132-2359 Yun Mi Shin https://orcid.org/0000-0001-9880-4004 Seong-Ju Kim https://orcid.org/0000-0003-1364-9960 Woo Dong Cho https://orcid.org/0000-0001-5366-778X Soyoung Irene Lee https://orcid.org/0000-0003-2473-2954

\section{REFERENCES}

1. American Psychiatric Association. Diagnostic and statistical manual of mental disorders. 5th ed. Washington, D.C: American Psychiatric Publishing;2013.

2. Rapport MD, Denney C, DuPaul GJ, Gardner MJ. Attention deficit disorder and methylphenidate: normalization rates, clinical effectiveness, and response prediction in 76 children. J Am Acad Child Adolesc Psychiatry 1994;33:882-893.

3. Gokcen C, Karadag M, Aksoy I. Methylphenidate induced lip and tongue biting. Clin Psychopharmacol Neurosci 2018; 16 : 218-220.

4. Su KP, Matsuoka Y, Pae CU. Omega-3 polyunsaturated fatty acids in prevention of mood and anxiety disorders. Clin Psychopharmacol Neurosci 2015;13:129-137.

5. Burgess JR, Stevens L, Zhang W, Peck L. Long-chain polyunsaturated fatty acids in children with attention-deficit hyperactivity disorder. Am J Clin Nutr 2000;71(1 Suppl):327S- 
3305 .

6. Gillies D, Sinn JKH, Lad SS, Leach MJ, Ross MJ. Polyunsaturated fatty acids (PUFA) for attention deficit hyperactivity disorder $(A D H D)$ in children and adolescents. Cochrane Database Syst Rev 2012:CD007986.

7. Bloch MH, Qawasmi A. Omega-3 fatty acid supplementation for the treatment of children with attention-deficit/hyperactivity disorder symptomatology: systematic review and meta-analysis. J Am Acad Child Adolesc Psychiatry 2011;50: 991-1000.

8. Sonuga-Barke EJ, Brandeis D, Cortese S, Daley D, Ferrin M, Holtmann $\mathrm{M}$, et al.; European ADHD Guidelines Group. Nompharmacological interventions for ADHD: systematic review and meta-analyses of randomized controlled trials of dietary and psychological treatments. Am J Psychiatry 2013; 170:275-289.

9. Kim HJ, Kim P, Shin CY. A comprehensive review of the therapeutic and pharmacological effects of ginseng and ginsenosides in central nervous system. J Ginseng Res 2013;37:8-29.

10. Itoh T, Zang YF, Murai S, Saito H. Effects of Panax ginseng root on the vertical and horizontal motor activities and on brain monoamine-related substances in mice. Planta Med 1989;55: 429-433.

11. Benishin CG. Actions of ginsenoside Rb1 on choline uptake in central cholinergic nerve endings. Neurochem Int 1992; 21:1-5.

12. Rudakewich M, Ba F, Benishin CG. Neurotrophic and neuroprotective actions of ginsenosides $R b(1)$ and $\mathrm{Rg}(1)$. Planta Med 2001;67:533-537.

13. Ko HJ, Kim I, Kim JB, Moon Y, Whang MC, Lee KM, et al. Effects of Korean red ginseng extract on behavior in children with symptoms of inattention and hyperactivity/impulsivity: a double-blind randomized placebo-controlled trial. I Child Adolesc Psychopharmacol 2014;24:501-508.

14. DuPaul GJ. Parent and teacher ratings of ADHD symptoms: psychometric properties in a community-based sample. J Clin Child Adolesc Psychol 1991;20:245-253.

15. So YK, Noh JS, Kim YS, Ko SG, Koh YJ. The reliability and validity of Korean parent and teacher ADHD Rating Scale. J Korean Neuropsychiatr Assoc 2002;41:283-289.
16. Busner J, Targum SD. The Clinical Global Impressions Scale: applying a research tool in clinical practice. Psychiatry (Edgmont) 2007:4:28-37.

17. Cho SZ, Chun SY, Hong KE, Shin MS. A study of the development and standardization of ADHD diagnostic system. J Korean Acad Child Adolesc Psychiatry 2000;11:91-99.

18. Kim HK. Assessment of memory disorders using Rey - Kim Memory Test. Korean J Rehabil Psychol 2001;8:29-48.

19. Kim HK. Kims EXIT (Executive Intelligence Test) for children: manual. Daegu:Neuropsychology Publishing Co.;2005.

20. Goodman D, Faraone SV, Adler LA, Dirks B, Hamdani M, Weisler $\mathrm{R}$. Interpreting $A D H D$ rating scale scores: linking $A D H D$ rating scale scores and $C G l$ levels in two randomized controlled trials of lisdexamfetamine dimesylate in $A D H D$. Prim Psychiatry 2010;17:44-52.

21. Vaisman N, Kaysar N, Zaruk-Adasha Y, Pelled D, Brichon G, Zwingelstein G, et al. Correlation between changes in blood fatty acid composition and visual sustained attention performance in children with inattention: effect of dietary n-3 fatty acids containing phospholipids. Am J Clin Nutr 2008;87: 1170-1180.

22. Lee SH, Park WS, Lim MH. Clinical effects of Korean red ginseng on attention deficit hyperactivity disorder in children: an observational study. J Ginseng Res 2011,35:226-234.

23. Portillo-Reyes V, Pérez-García M, Loya-Méndez Y, Puente AE. Clinical significance of neuropsychological improvement after supplementation with omega-3 in 8-12 years old malnourished Mexican children: a randomized, double-blind, placebo and treatment clinical trial. Res Dev Disabil 2014;35: 861-870.

24. Muthayya S, Eilander A, Transler C, Thomas T, van der Knaap $\mathrm{HC}$, Srinivasan K, et al. Effect of fortification with multiple micronutrients and $n-3$ fatty acids on growth and cognitive performance in Indian schoolchildren: the CHAMPION (Children's Health and Mental Performance Influenced by Optimal Nutrition) Study. Am J Clin Nutr 2009;89:1766-1775.

25. Hirayama S, Hamazaki T, Terasawa K. Effect of docosahexaenoic acid-containing food administration on symptoms of attention-deficit/hyperactivity disorder - a placebo-controlled double-blind study. Eur J Clin Nutr 2004;58:467-473. 